Supplement of Ocean Sci., 15, 1399-1418, 2019

https://doi.org/10.5194/os-15-1399-2019-supplement

(C) Author(s) 2019. This work is distributed under

the Creative Commons Attribution 4.0 License.

(c) (1)

Supplement of

\title{
Extreme sea levels in the Baltic Sea under climate change scenarios - Part 1: Model validation and sensitivity
}

\section{Christian Dieterich et al.}

Correspondence to: Christian Dieterich (christian.dieterich@smhi.se)

The copyright of individual parts of the supplement might differ from the CC BY 4.0 License. 


\section{Model Sensitivity}

The figures shown in this supplementary material are discussed in the main article in section 4.
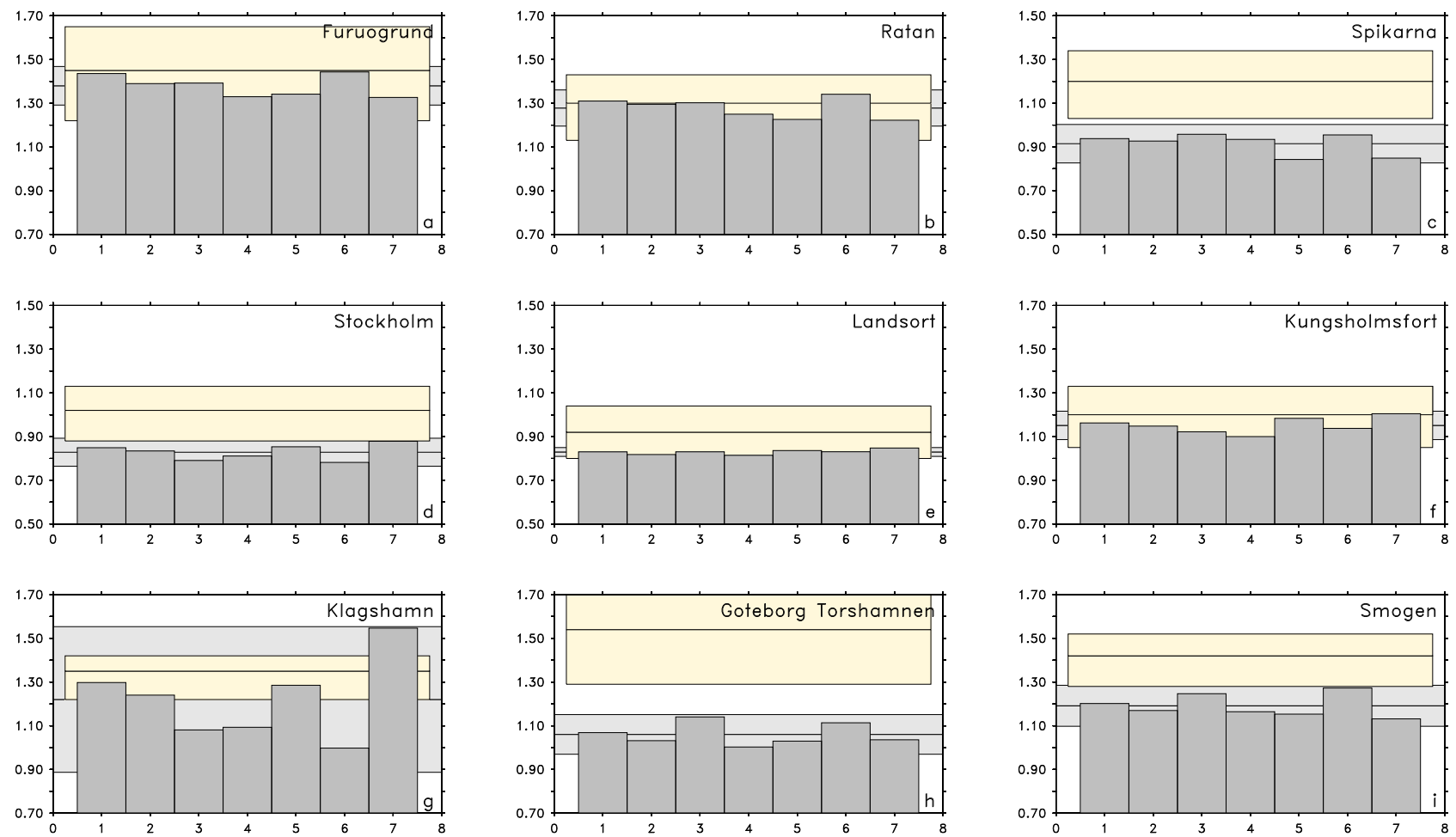

Figure S1. Sensitivity of ESLs using different analysis periods. Extreme sea levels (m) from RCA4-NEMO ERA40 with 100-year return period for the same stations as in Fig. 2. The different analysis periods are 1961 to 2005 (1), 1970 to 2009 (2), 1961 to 1989 (3), 1970 to 1999 (4), 1980 to 2009 (5), 1961 to 1984 (6) and 1985 to 2009 (7). The horizontal line represents the mean of the different estimates and the lightly shaded area around it is the $95 \%$ confidence interval. 

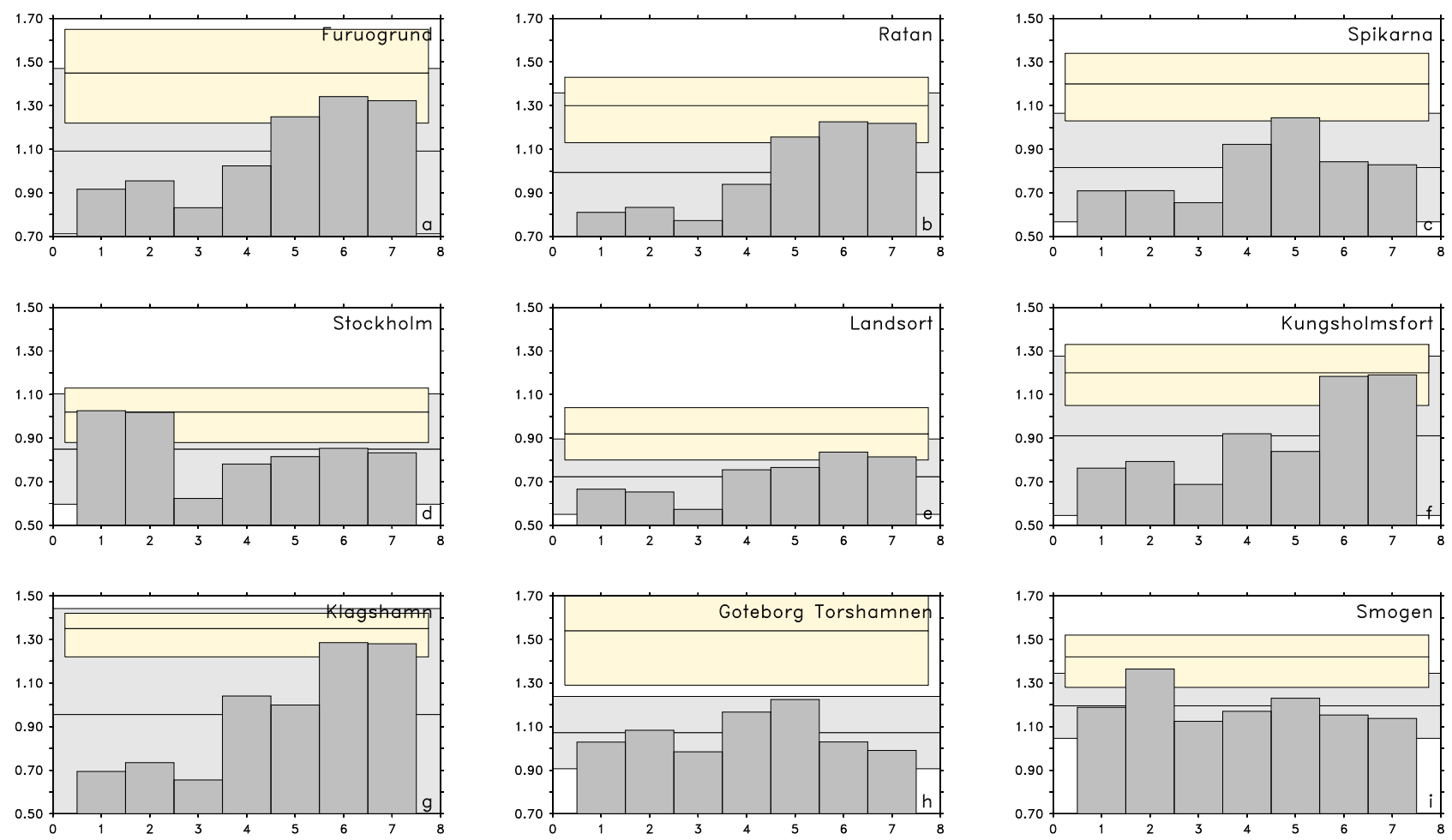

Figure S2. Sensitivity of ESLs using different atmospheric forcings. Extreme sea levels (m) as in Fig. S1. The different sensitivity runs are NEMO-Nordic ERA40 (1), NEMO-Nordic ERA-interim (2), NEMO-Nordic interpolated (3), NEMO-Nordic 3.6 ERA40 (4), NEMO-Nordic 3.6 EURO4M (5), RCA4-NEMO ERA40 (6), RCA4-NEMO-1hr ERA40 (7). Table 5 gives an overview of the experiments. The common analysis period was 1980 to 2009, except for NEMO-Nordic 3.6 ERA40 it was 1980 to 2005. 

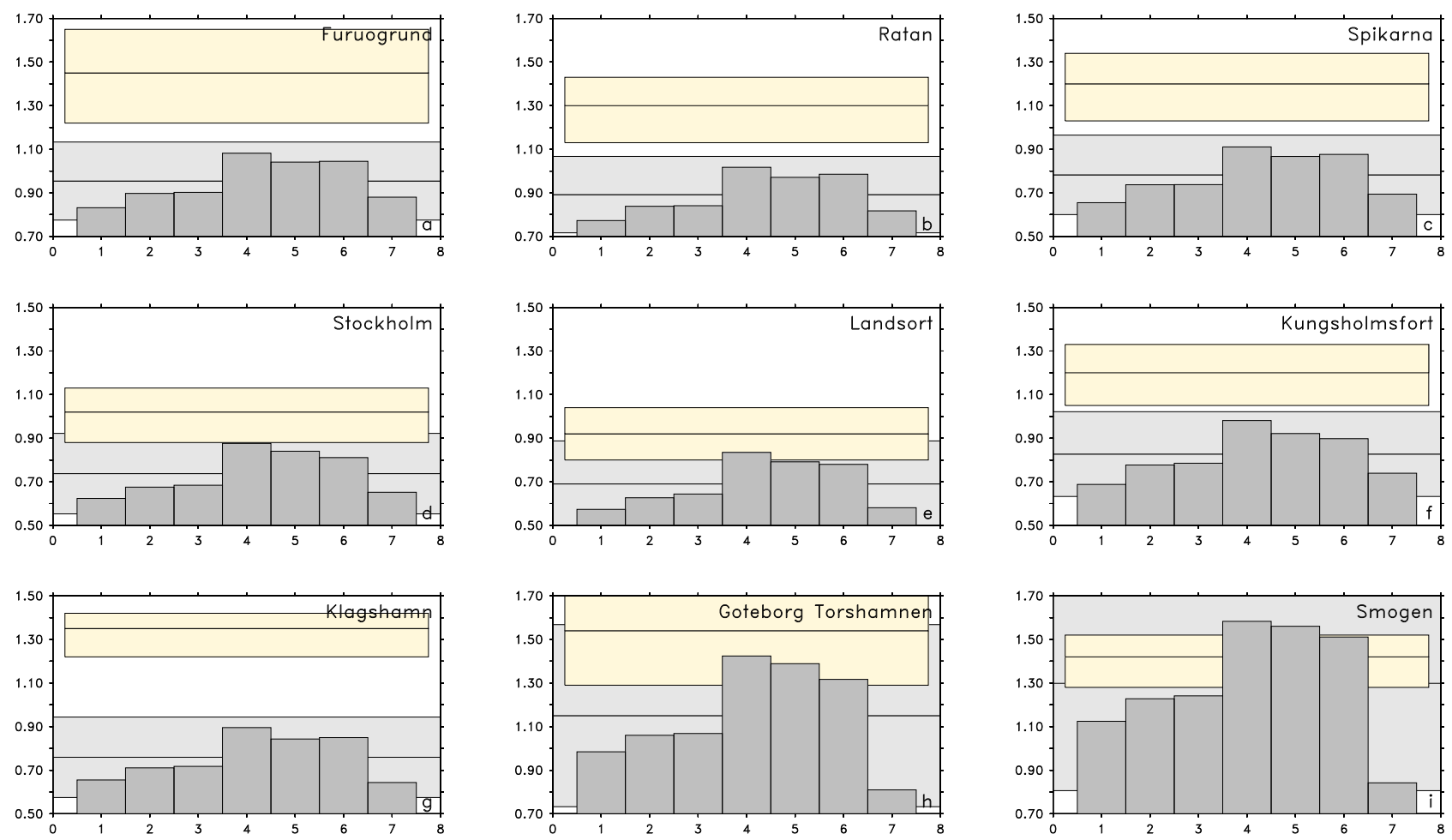

Figure S3. Sensitivity of ESLs using different open boundary conditions. Extreme sea levels (m) as in Fig. S1. The different bars refer to the experiments NEMO-Nordic interpolated (1), NEMO-Nordic ORAS4 (2), NEMO-Nordic ORAS4 b (3), NEMO-Nordic ORAS4 c (4), NEMO-Nordic Surge (5), NEMO-Nordic Surge b (6), NEMO-Nordic NOT (7). The different experiments are listed in Table 6. The common analysis period was 1980 to 2009 . 

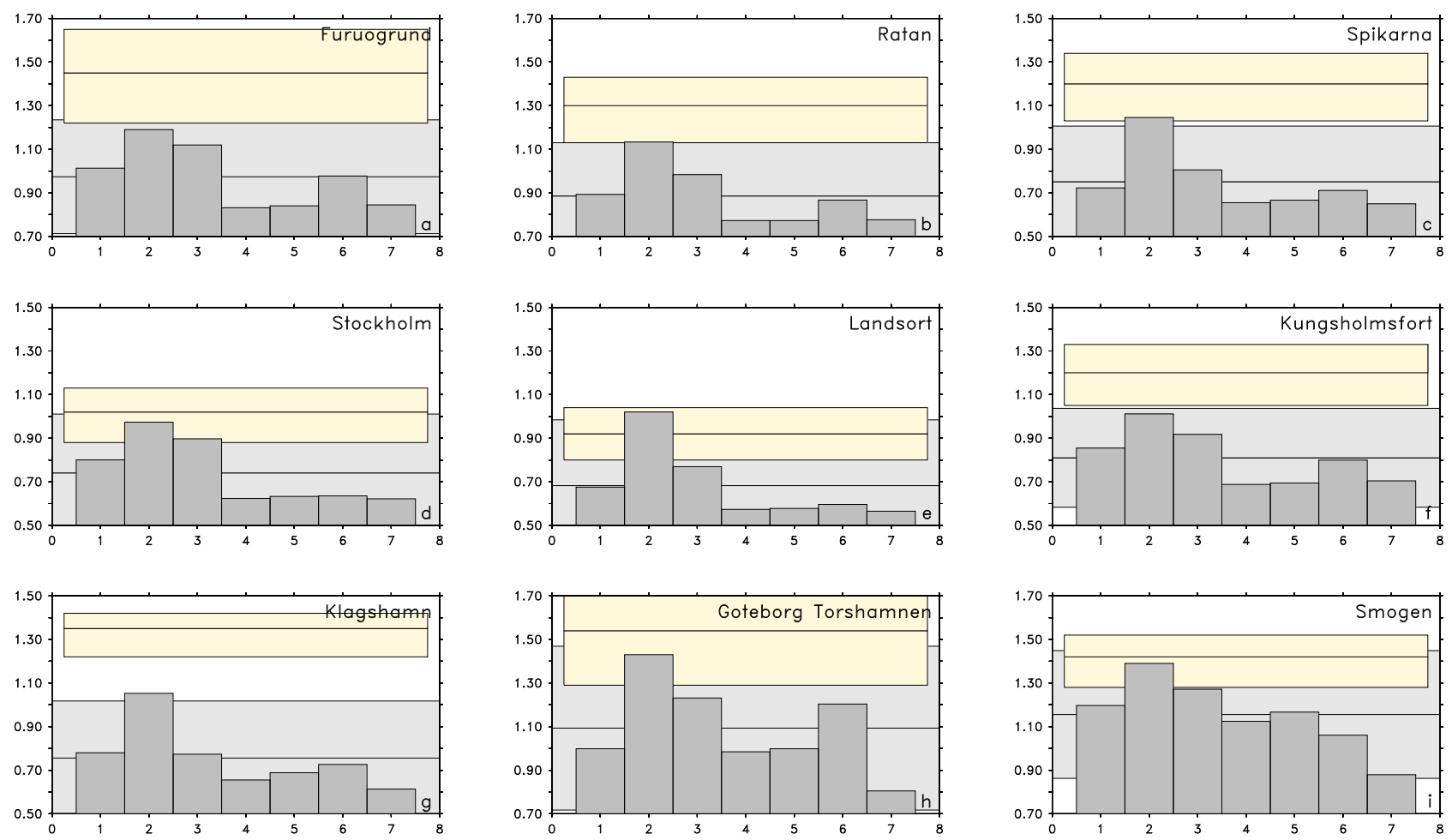

Figure S4. Sensitivity of ESLs varying miscellaneous model parameters. Extreme sea levels (m) as in Fig. S1. The different sensitivity runs are NEMO-Nordic viscous (1), NEMO-Nordic no-slip (2), NEMO-Nordic free-slip (3), NEMO-Nordic interpolated (4), NEMO-Nordic MSL (5), NEMO-Nordic E-HYPE (6), NEMO-Nordic discharge (7). Table 7 gives an overview of the experiments. The common analysis period was 1980 to 2009 . 